
\title{
25 Research Soure \\ Locking plate for cubitus varus correction in osteogenesis imperfecta: a case report of 7 year-old girl
}

\section{Pan Hong}

Wuhan Union Hospital

Ruikang Liu

Huazhong University of Science and Technology Tongji Medical College

Saroj Rai

National Academy of Medical Sciences

Ruijing Xu

Wuhan Union Hospital

Jin Li ( $\sim$ lijin2003whxh@foxmail.com )

Wuhan Union Hospital https://orcid.org/0000-0003-0610-2991

\section{Research}

Keywords: Cubitus varus, osteogenesis imperfecta, locking plate

Posted Date: July 17th, 2020

DOI: https://doi.org/10.21203/rs.3.rs-41740/v1

License: (c) (1) This work is licensed under a Creative Commons Attribution 4.0 International License. Read Full License

Version of Record: A version of this preprint was published at Frontiers in Pediatrics on January 12th, 2022. See the published version at https://doi.org/10.3389/fped.2021.781703. 


\section{Abstract \\ Background}

Cubitus varus deformity is a common complication of supracondylar fractures in children. However, cubitus varus in osteogenesis imperfect $(\mathrm{OI})$ children is a rare but challenging situation. To the author's knowledge, it is the first study discussing the correction of cubitus varus deformity in OI patient. Here we report a case of an 7-year-old OI girl who presented with varus deformities of the bilateral lower extremities and cubitus varus deformity of the left elbow due to a supracondylar fracture of humerus 3 year ago. The patient's parent gave a history of supracondylar fracture of left humerus 3 years ago. Without medical intervention, the patient was admitted into our institution for corrective surgery with the diagnosis of osteogenesis imperfecta and cubitus varus deformity in the left arm.

\section{Result}

Medications including calcium, vitamin D and bisphosphonates were administered before the corrective surgery of cubitus varus, and a single locking plate was used to after the osteotomy. Right after the surgery, the appearance and ROM of the left arm was almost normal. Combined with gradual rehabilitation, the ROM of the left arm was normal without pain during daily use. The hardware was removed as the nailing of the forearm fractures was performed at the same time.

\section{Conclusion}

Cubitus varus is a common deformity in children resulting from elbow injuries, but it presents a challenging situation in OI patients. Locking plate combined with meticulous pharmacological intervention provides a good option for corrective surgery of cubitus varus in Ol.

\section{Introduction}

Cubitus varus is a common complication of supracondylar fractures in children ${ }^{[1]}$. The surgical interventions for this deformity remain controversial. Many techniques of humeral osteotomy has been reported to correct this deformity in order to avoid later complications including ulnar nerve palsy[2], posterolateral rotatory instability ${ }^{[3]}$, secondary distal humeral fracture ${ }^{[4]}$. However, report on correction of cubitus varus in osteogenesis imperfect $(\mathrm{OI})$ patients is rare.

Osteogenesis imperfecta (OI), also known as "brittle bone disease", is a heritable collagen-related disordersis, which is characterized by a dysfunction of the connective tissue due to mutations in the genes COLIA and COL1A2 that codify for the type 1 collagen chains ${ }^{[5]}$. Patients with Ol frequently manifest unintentional fracture, growth retardation, and deformity due to bone fragility. Ol occurs in about 1 in $10,000-20,000$ live births $^{[6]}$, but the incidence might be higher because of its heterogeneity ${ }^{[7]}$. Due to 
poor bone quality, children with OI experience high rates of fractures. The treatment of such fractures is quite difficult, and the treatment of post-traumatic deformity is even more challenging. Here, we presented a case of cubitus varus in a seven year-old girl with OI The elbow deformity was successfully treated by closing-wedge osteotomy fixated by a locking plate.

\section{Case Presentation}

A 7 year-old girl with bilateral femoral deformity and fresh bilateral femoral shaft fracture was presented in our clinic. During her early childhood, the patient frequently sustained multiple fractures on the lower extremities by petty violence. At a municipal hospital, she was suspected as Ol. However, no medical therapy was administered for her thereafter. On physical examination, the cubitus varus of left elbow was noticed with a history of supracondylar humeral fracture (SCHF) 3 years ago. The patient received closed reduction and external fixation for a Gartland type III SCHF when she was 4 years old (see Fig. 1). However, the diagnosis of $\mathrm{Ol}$ was still not mentioned in the discharge paper after the first surgery. No supplemental drugs including calcium, vitamin $D$ and bisphosphonates were given to this patient to increase her bone mass after the SCHF surgery. Fortunately, according to her parents, no elbow fractures happened afterwards.

Dual-energy X-ray absorptiometry (DXA) is the gold standard for measuring bone mineral density (BMD), making the diagnosis of osteoporosis, monitoring BMD, and is used in the calculation of fracture risk ${ }^{[8]}$. During her hospital stay at our institute, DXA came up with a $Z$ value -5 , suggesting serious osteoporosis. Multiple osteotomies and telescoping rods were used to fixate the lower extremity of this patient (see Fig. 2). Because of high rate of non-union and possible failure of hardware, the cubitus varus was scheduled to be corrected later. Calcium and vitamin D were given to patient immediately after rodding. Oral bisphosphonates were administered one month later after the rodding surgery. Intravenous bisphosphonates were not available at our institute.

The patient was admitted into our institute 8 months after rodding surgery for cubitus varus correction. The patient presented approximately 30 degrees cubitus varus with prominent lateral condyle and her contralateral elbow demonstrated approximately 6 degrees valgus. Our surgical plan was simple lateral wedge osteotomy with slight medial translation of distal part of the humerus. Mechanical problems with Kirschner wires, external fixator and intramedullary nail in achieving optimal fixation of the supracondylar osteotomy are poor bone density, as well as significant translational and rotational force in this region. Therefore, a $3.5 \mathrm{~mm}$ locking reconstruction plate was used (see Fig. 3). After the surgery, a long-arm cast was applied for 4 weeks. The oral bisphosphonates was discontinued for three months. At one-month follow-up, the patient demonstrated full elbow motion without pain, and X-ray images demonstrated early healing. At three-month follow-up, the patient displayed uneventful healing. The callus seemed fine and oral bisphosphonates was resumed. The DXA indicated $Z$ value -3 , suggesting increased BMD but still osteoporosis. The hardware was not prominent on the lateral condyle, and the plate was scheduled to be removed 12 months later. Mayo elbow performance score (MEPS) ${ }^{[9]}$ was used for patient to assess her over-all effectiveness of treatment, and the result was excellent (95 points). 
Eleven months after the corrective surgery, the patient came back to our institute with left radius fracture. Elastic stable intramedullary nail (ESIN) was used to fixate the radius and ulna. Besides, plate removal was performed at the same time (see Fig. 4).

All procedures performed in this study were performed in accordance with the ethical standards of the national research committee and the 1964 Helsinki declaration and its later amendments or comparable ethical standards. The patient's legal guardian provided informed consent for publication of the case.

\section{Discussion}

Telescoping intramedullary fixation is the standard treatment strategy for long bone fractures in $\mathrm{Ol}$ especially in lower extremities ${ }^{[10]}$. Therefore, telescoping rods were used for bilateral femoral deformity and fresh bilateral femoral shaft fracture in this study. However, as for this patient requiring humerus deformity correction, telescoping rodding was not a suitable choice. There are many fixations methods regarding the correction of cubitus varus including Kirschner wire/pin fixation, tension-band wiring, screws alone, external fixator, plating. However, routine use of K-wire or external fixator might not provide enough stability in osteoporotic bone, especially in this cubitus varus case, $\mathrm{K}$-wire or external fixator could hardly provide sufficient stability.

Osteoporotic fractures represent unique fixation challenges related to decreased BMD and higher rates of screw pullout, but locked plating creates a fixed-angle construct with great resistance to failure in osteoporotic bone with thin cortices ${ }^{[11]}$. Hanke et al $^{[12]}$ reported that locking plate technique was successful as a salvage procedure in a case report of femoral fracture in OI. There were also other reports advocating locking plate in osteoporotic fractures and non-union of humerus in $\mathrm{Ol}^{[13-16]}$. Therefore, in this case, a $3.5 \mathrm{~mm}$ locking plate was used to fixate the osteotomy site. Surgical fixation of the pathological bones of patients with $\mathrm{OI}$ should be intramedullary in nature, which avoids the stress risers created by a stand-alone plate and screw construct ${ }^{[17]}$. The advantage of locking plate is the screws with angular stability are evenly distributed throughout the loading between the bone and screws. There was report about dual-plating ${ }^{[14]}$, but in this case a single lateral plate was sufficient.

There is no gold standard surgical correction for cubitus varus, and many types of osteotomies have been reported including lateral wedge osteotomies, step-cut, dome osteotomies, and spatial correction using Taylor spatial frame (TSF) ${ }^{[18]}$. Considering operability and reproducibility, we choose lateral wedge osteotomies. For this patient, lateral closing wedge osteotomy combined with slight medial translation of distal part of humerus was able to correct the varus deformity without residual lateral condylar prominence.

Pharmaceutical intervention plays an indispensable part for postoperative recovery. Bisphosphonates inhibit bone resorption by reducing the number and activity of osteoclasts: this results in improved vertebrae form and density, increased cortical diameter and increased bone volume ${ }^{[19]}$. Bisphosphonate therapy has been given to children with OI over three decades and various studies had concluded that 
bisphosphonates could increase BMD in children with OI and decrease their fracture rate in the range of 30 to $60 \%{ }^{[20-22]}$. Recent studies showed both oral and intravenous bisphosphonate therapy seemed to be associated with a lower rate of long-bone fractures in children with $0 \mathrm{I}^{[22,28]}$. Trejo et al ${ }^{[28]}$ suggested intravenous administration might be a better choice that it could improve mobility and had a positive effect on the spine of growing children with Ol. However, intravenous bisphosphonates was unavailable at our institute.

Non-union is a known complication in patients with Ol, with or without the application of bisphosphonates treatment ${ }^{[23,24]}$. Whether bisphosphonates treatment affects the healing of fractures in Ol remains controversial ${ }^{[25,26]}$. However, there were reports of non-union in the humerus ${ }^{[13,14,23,25]}$, and non-union in the upper extremity can be quite disabling ${ }^{[27]}$. Therefore, concerning the low BMD, we postponed the corrective surgery for more than 6 months, and calcium and vitamin D supplements were used immediately after rodding surgery to increase the patient's BMD. Subsequently, bisphosphonates were applied to the patient one month later. However, concerning the possible impact on postoperative healing of frature, oral administration of bisphosphonates was discontinued after elbow corrective surgery and resumed after 3-month follow up. Fortunately, this patient displayed an uneventful healing process without any adverse event including non-union.

There were several limitations in our study. Firstly, intravenous bisphosphonates was unavailable in our hospital, and intravenous bisphosphonate might resulted in better BMD and clinical performance.

Besides, anatomic locking plate might be a better choice for corrective surgery if available. Futhermore, cost-effective analysis using locking plate remains to be investigated.

\section{Conclusion}

Locking plate combined with meticulous pharmacological intervention delivers good clinical result for corrective surgery of cubitus varus in pediatric patient with osteogenesis imperfecta.

\section{Abbreviations}

Osteogenesis imperfecta $=\mathrm{Ol}$; supracondylar humeral fracture $=$ SCHF; dual energy $\mathrm{x}$-ray absorptiometry = DXA; Elastic stable intramedullary nail = ESIN; bone mineral density $=\mathrm{BMD}$; Taylor spatial frame $=\mathrm{TSF}$; Mayo elbow performance score $=$ MEPS.

\section{Declarations}

\section{Acknowledgement}

Not applicable 


\section{Availability of data and materials:}

Data sharing is not applicable to this article as no datasets were generated or analyzed during the current study.

\section{Ethics approval and consent to participate:}

This study was approved by the Ethics Committee of Tongji Medical College, Huazhong University of Science and Technology. The parents have signed an informed consent allowing further research.

\section{Consent for publication:}

Written consents were obtained from the legal guardians of patient for publication of this paper.

\section{Competing interests:}

The authors declare that they have no competing interests.

\section{Funding:}

This study was supported by Nature Science Foundation of Hubei Province [2018CFB590] and Wei Ye Pharmaceutical industry (grant number 02.01.0475). The authors had full editorial control over the results presented here and interpretation of the findings.

\section{Authors' contributions:}

$\mathrm{JL}^{*}$ is in charge of the main idea and is the guarantor of integrity of the entire study; $\mathrm{PH}$ and RKL are in charge of the study concepts, design, manuscript preparation and editing; $\mathrm{PH}$ and SR are in charge of the language polishing and the grammar revision. All authors read and approved the final manuscript.

\section{References}

1. Davids JR, Lamoreaux DC, Booker RC, et al. Translation step-cut osteotomy for treatment of post traumatic cubitus varus. J Pediatr Orthop. 2011;31(4):353 - 65.

2. Abe $M$, Ishizu $T$, Shirai $H$, et al. Tardy ulnar nerve palsy caused by cubitus varus deformity. J Hand Surg Am. 1995;20:5-9.

3. O'Driscoll SW, Spinner RJ, McKee MD, et al. Tardy posterolateral rotatory instability of the elbow due to cubitus varus. J Bone Joint Surg Am. 2001;83:1358-69.

4. Park HW, Yang IH, Joo SY, et al. Refractures of the upper extremity in children. Yonsei Med J. 2007; $48: 255-60$. 
5. Rauch F. Glorieux FH. Osteogenesis imperfecta. Lancet. 2004;363(9418):1377-85.

6. Monti $E$, Mottes $M$, Fraschini $P$, et al. Current and emerging treatments for the management of osteogenesis imperfecta. Ther Clin Risk Manag. 2010;6:367 - 81.

7. Plotkin H. Syndromes with brittle bones: hyperostotic bone disease and fibrous dysplasia of bone. Pediatric Endocrinology. 2007;5(2):559 - 80.

8. Morgan SL. Prater GL. Quality in dual-energy X-ray absorptiometry scans. Bone. 2017;104:13-28.

9. Morrey BF. An KN, Chao EYS. Functional Evaluation of the Elbow. In: Morrey BF, ed. The Elbow and its Disorders. Philadelphia: W.B. Saunders;1993: pp. 86-97.

10. Jaenne MF, Suken AS, Maegen JW, et al. Osteogenesis Imperfecta: a pediatric orthopedic perspective. Orthop Clin N Am. 2019;50:193-209.

11. Graham JD, Patric JK, Justin MH. Locked plating and advanced augmentation techniques in osteoporotic fractures. Orthop Clin North Am. 2019;50(2):159 - 69.

12. Hanke HS, Keel MJ, Todorski IA, et al. The reversed less invasive stabilisation system-distal femur technique: Application in an adult patient with osteogenesis imperfecta sustaining a femoral fracture. J Orthop Case Rep. 2017;7:71 - 5.

13. Sano S, Oe K, Fukui T, et al. Humeral Shaft Nonunion in a Patient with Osteogenesis Imperfecta Treated with Mandible Locking Plate Fixation: A Case Report. J Orthop Case Rep. 2019;9(3):19-21.

14. Mark SH, Matthew AM, Paul WE, et al. Distal humeral atrophic nonunion in a child with osteogenesis imperfecta. J Pediatr Orthop. 2013;33(7):725-9.

15. Miranda MA. Locking plate technology and its role in osteoporotic fractures. Injury. 2007;38(3):35 9.

16. Cornell CN, Ayalon O. Evidence for success with locking plates for fragility fractures. HSS J. $2017 ; 7: 164-9$.

17. Enright WJ, Noonan KJ. Bone plating in patients with type III osteogenesis imperfecta: results and complications. lowa Orthop J. 2006;26:37-40.

18. Andrea SB, Brian P, Lisa LL. Surgical correction of cubitus varus. J Hand Surg Am. 2016;41(3):447 52.

19. Sánchez-Sánchez LM. Cabrera-Pedroza AU, Palacios-Saucedo G. de la Fuente-Cortez B. [Zoledronic acid (zoledronate) in children with osteogenesis imperfecta]. Gac Med Mex. 2015;151(2):164-8.

20. Devogelaer JP. Malghem J, Maldague B. Nagant de Deuxchaisnes C. Radiological manifestations of bisphosphonate treatment with APD in a child suffering from osteogenesis imperfecta. Skeletal Radiol. 1987: 16,360-363.

21. Trejo P. Rauch F. Osteogenesis imperfecta in children and adolescents-new developments in diagnosis and treatment. Osteoporos Int. 2016;27(12):3427-37.

22. Dwan K, Phillipi CA, Steiner RD, et al. Bisphosphonate therapy for osteogenesis imperfecta. Cochrane Database Syst Rev. 2016. 
23. Gamble JG, Rinsky LA, Strudwick J, et al. Nonunion of fractures in children who have osteogenesis imperfecta. J Bone Joint Surg Am. 1988;70A:439-43.

24. Agarwal V, Joseph B. Non-union in osteogenesis imperfecta. J Pediatric Orthop B. 2005;14:451-5.

25. Munns CF, Rauch F, Zeitlin L, et al. Delayed osteotomy but not fracture healing in pediatric osteogenesis imperfecta patients receiving pamidronate. J Bone Miner Res. 2004;19:1779-86.

26. Glorieux FH. Experience with bisphosphonates in osteogenesis imperfecta. Pediatrics. 2007;199(2): 163-5.

27. Amako M, Fassier F, Hamdy RC, et al. Functional analysis of upper limb deformities in osteogenesis imperfect. J Pediatr Orthop. 2004;24:689-94.

28. Trejo P. Rauch F. Osteogenesis imperfecta in children and adolescents-new developments in diagnosis and treatment[J]. Osteoporos Int. 2016;27(12):3427-37.

\section{Figures}




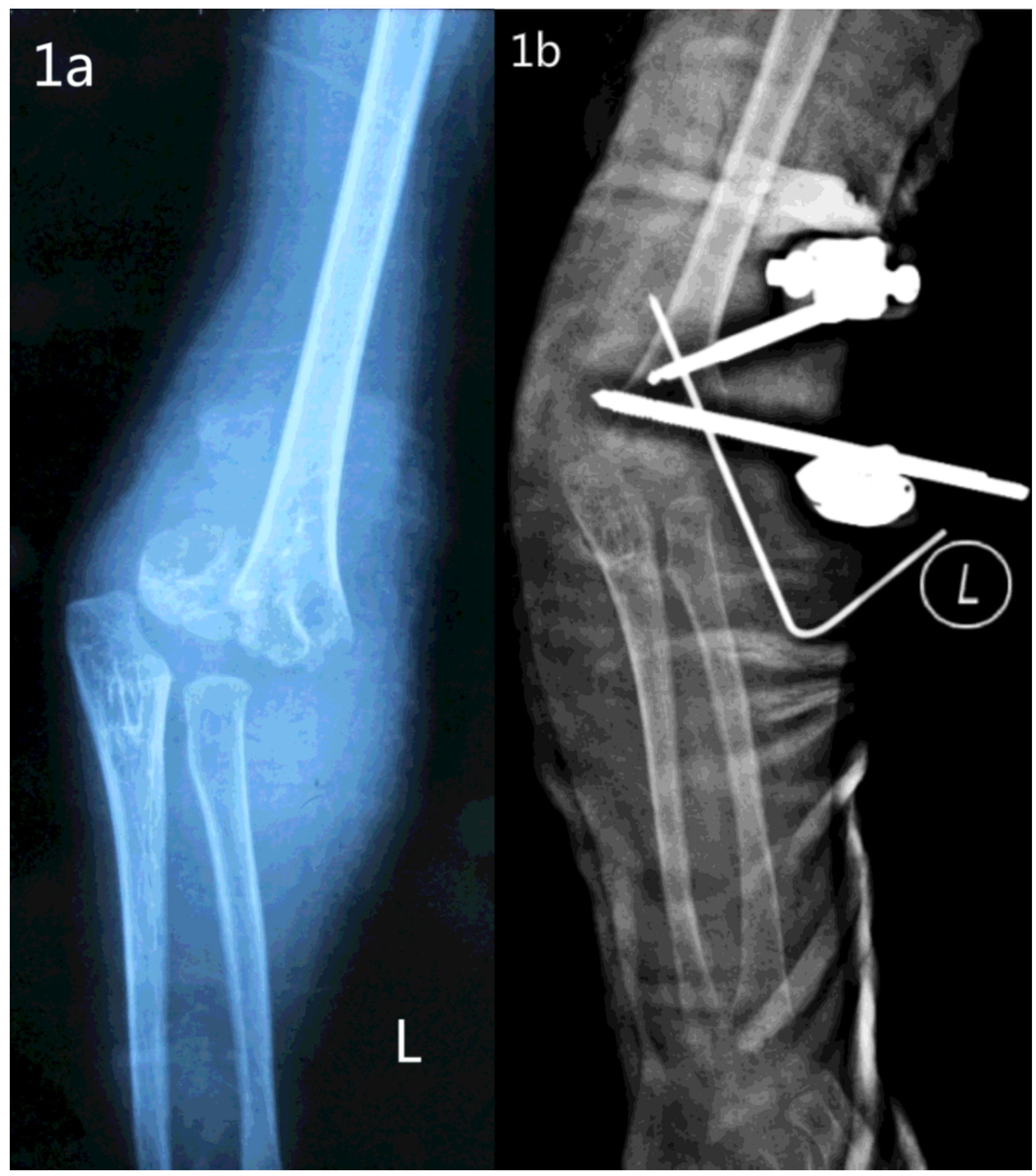

\section{Figure 1}

Gartland type III supracondylar fracture of a 4 year-old girl a. AP view of Gartland type III supracondylar fracture b. AP view of postoperative elbow 


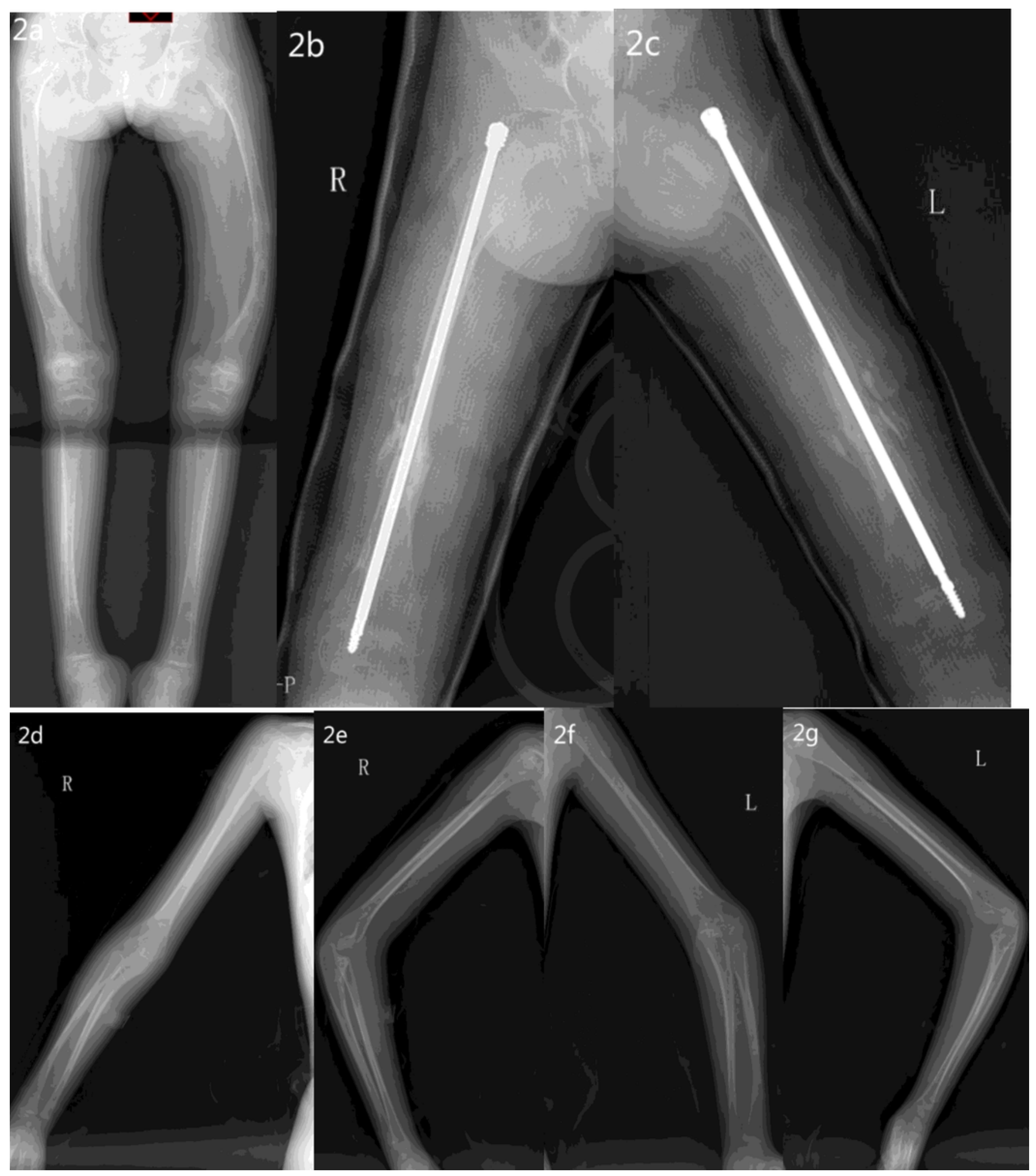

\section{Figure 2}

Radiograph of 7 year-old girl a. Full-length image of the lower extremities showing fracture and bowing in bilateral femur b. Nailing of the right femur c. Nailing of the left femur d. AP view of the right elbow e. Lateral view of the right elbow $\mathrm{f}$. AP view of the left elbow $\mathrm{g}$. Lateral view of the left elbow 


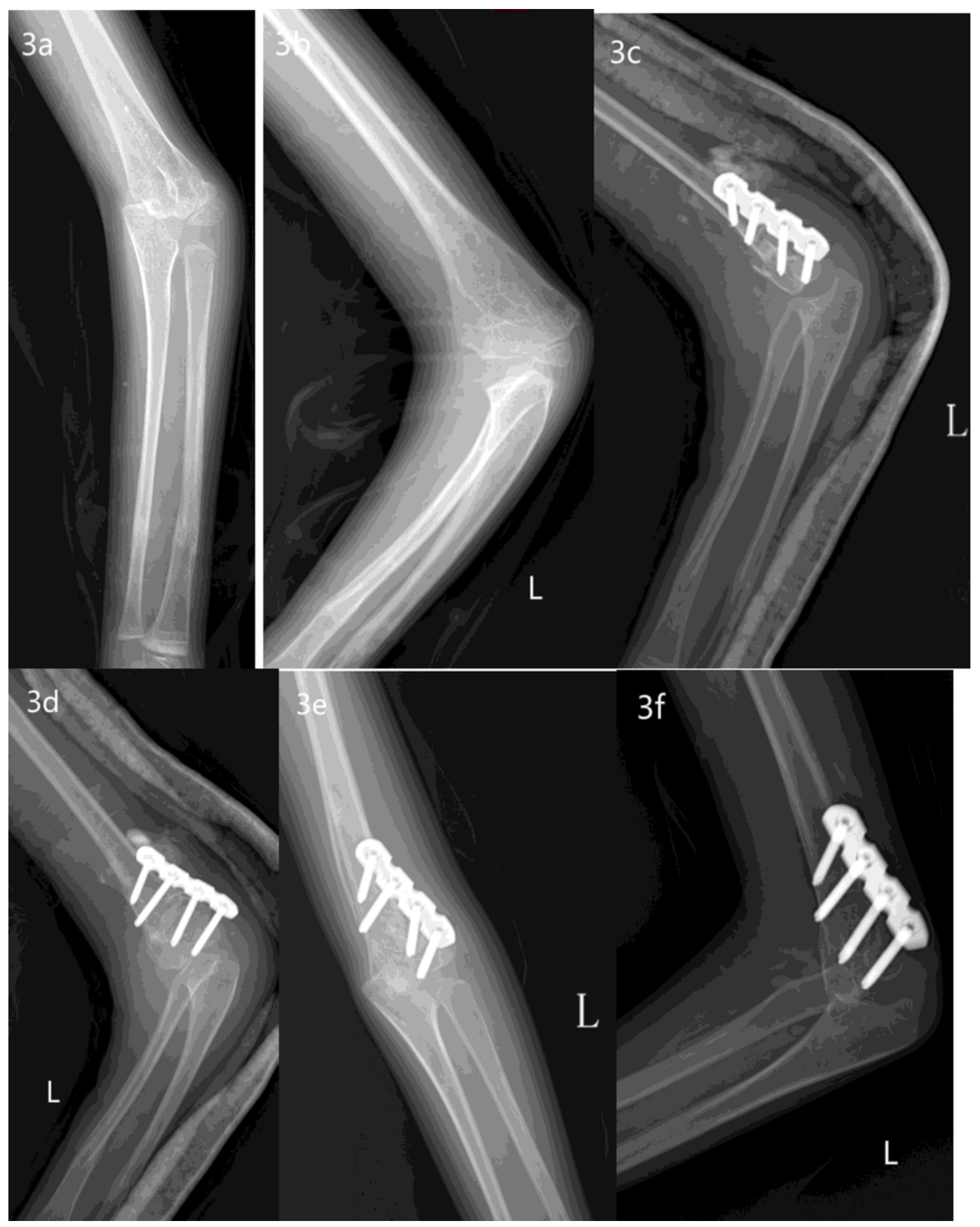

\section{Figure 3}

Correction of cubitus varus a. Preoperative AP view of left elbow b. Preoperative lateral view of left elbow c. Postoperative lateral view of the left elbow d. Postoperative AP view of the left elbow e. AP view of the left elbow at 3rd month follow-up f. Lateral view of the left elbow at 3rd month follow-up 


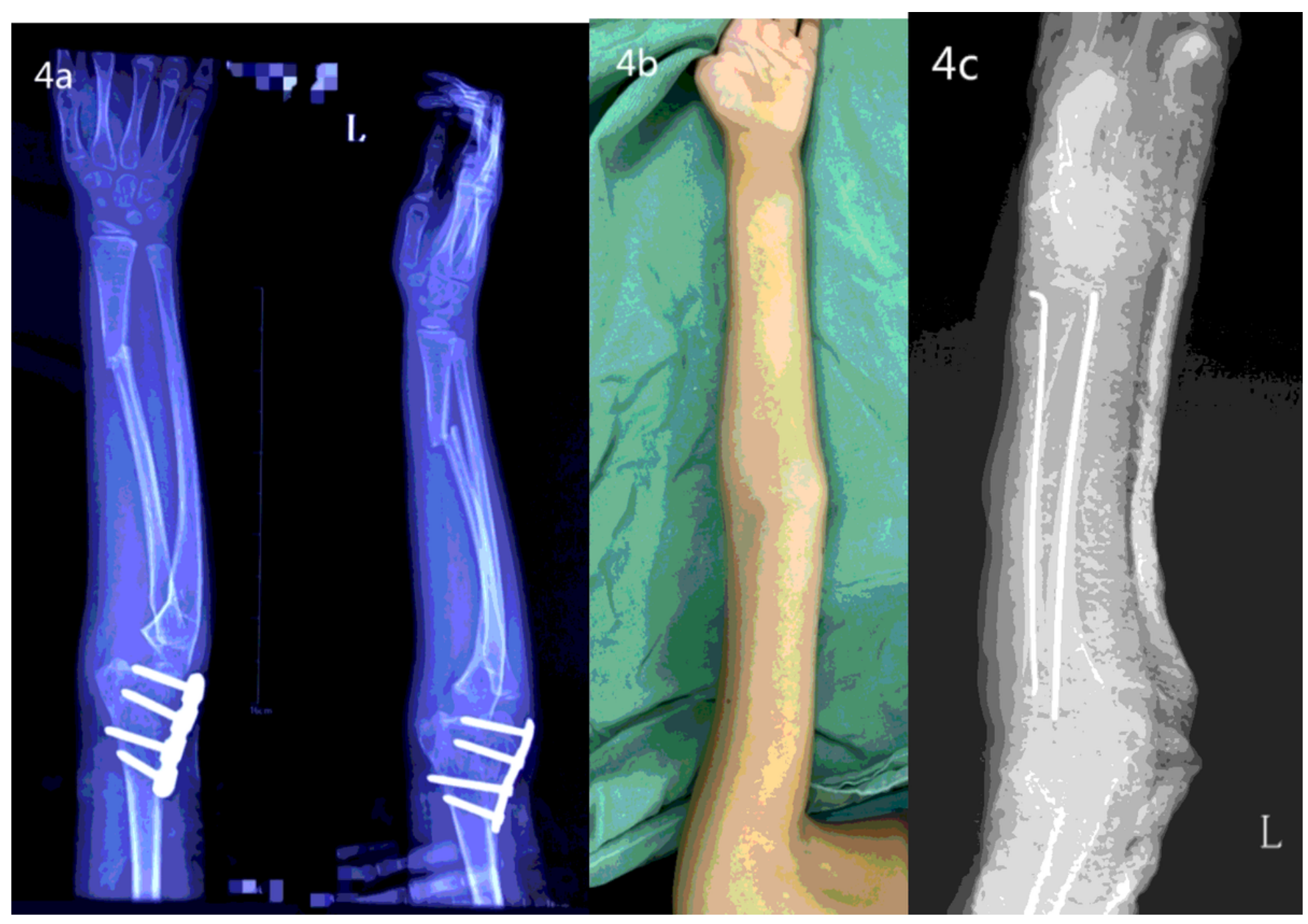

Figure 4

Removal of the plate and nailing of the forearm a. Image of the distal radial fracture b. Appearance of left elbow before removing the plate c. Intramedullary nailing of the left forearm

\section{Supplementary Files}

This is a list of supplementary files associated with this preprint. Click to download.

- checklistforcasereport6.docx 